\title{
INTRODUCCIÓN AL USO DE COAGULANTES NATURALES EN LOS PROCESOS DE POTABILIZACIÓN DEL AGUA
}

\section{INTRODUCTION TO NATURAL COAGULANTS AND THEIR USE IN WATER PURIFICATION PROCESSES}

\author{
ORTEGA, A. ${ }^{1}$; CACERES, L. ${ }^{2}$; CASTIBLANCO, L. ${ }^{3}$ \\ ${ }^{1}$ MSC. Angie Ortega Ramírez. Profesor Titular, Programa de \\ Ingeniería Química, Facultad de Ingenierías, Universidad de \\ América, e-mail: Angie.Ortega@profesores.uamerica.edu.co. \\ ${ }^{2}$ BSc. Luis Cáceres Durán, Programa de Ingeniería Química, \\ Facultad de Ingenierías, Universidad de América, e-mail: \\ Luis.Caceres2@estudiantes.uamerica.edu.co. \\ ${ }^{3}$ BSc. Laura Castiblanco Molina. Programa de Ingeniería \\ Química, Facultad de Ingenierías, Universidad de América, e-mail: \\ Laura.Castiblanco@estudiantes.uamerica.edu.co. \\ Universidad de América \\ Fundación Universitaria. BOGOTÁ D.C, Colombia. \\ Tel: 57-1-3376680, Fax: 57-1-3362941
}

\begin{abstract}
Resumen
El acceso al recurso hídrico es una de las necesidades básicas para la supervivencia de los seres humanos y animales, por ende, es necesario buscar estrategias y procesos que mejoren la calidad del agua potable, basada en procesos sustentables, amigables con el medio ambiente y seguro para el consumo humano. Por tanto, el presente artículo realiza una revisión detallada del uso de coagulantes naturales en el tratamiento de agua potable con el propósito de reemplazar los coagulantes químicos, mediante el uso de base de datos académicas y científicas, se encuentra que los aglomerantes son a base de bacterias, animales y plantas como la Opuntia Ficus Indica, y entre otros coagulantes que son viables para presentarse como alternativas naturales en el proceso de coagulación para la potabilización del recurso hídrico.
\end{abstract}

Palabras clave: Agua potable, gestión de recursos hídricos, procesos químicos.

\begin{abstract}
Access to water resources is one of the basic needs for the survival of humans and animals, therefore, it is necessary to seek strategies and processes that improve the quality of drinking water, based on sustainable, environmentally friendly and safe processes. for human consumption. Therefore, this article makes a detailed review of the use of natural coagulants in the treatment of drinking water with the purpose of replacing chemical coagulants, through the use of academic and scientific databases, it is found that the binders are based on bacteria, animals and plants such as Opuntia Ficus Indica, and among other coagulants that are viable to present themselves as natural alternatives in the coagulation process for the purification of water resources.
\end{abstract}

Keywords: Drinking water, water resources management, chemical processes. 


\section{INTRODUCCIÓN}

El agua es un recurso natural limitado y un bien público fundamental para la vida y la salud, aproximadamente el $75 \%$ de agua en el planeta está en forma líquida, gaseosa y congelada, sin embargo, el $95 \%$ es agua salada y solo $0.01 \%$ es agua dulce en ríos o lagos,(Odlare, 2014) El derecho humano al agua es indispensable para vivir dignamente y es condición previa para la realización de otros derechos humanos.(Tejado Gallegos \& Olmos Pérez, 2014) La importancia de una fuente confiable de agua es vital para el desarrollo sostenible de una sociedad. El crecimiento demográfico y el incremento de los estándares de vida están ejerciendo una presión sin precedentes en las reservas de agua(Food and Agriculture Organization of the United Nations, 2013). Para el 2050, se espera que 1800 millones de personas vivan en áreas con extrema escasez de agua $(<500$ $\mathrm{m}^{3}$ por año per capita), y dos tercios de la población mundial podría estar bajo condiciones de "alto estrés" (Entre 500 y $1000 \mathrm{~m}^{3}$ por año per capita). (Damkjaer \& Taylor, 2017). El no tener acceso a agua potable ocasiona anomalías en el cuerpo humano como diarrea, la cual, es la segunda causa de muerte más común en niños con menos de 5 años en países en desarrollo. Los niños de comunidades pobres y remotas sin acceso a agua potable son la población de mayor riesgo («Una Ryan», 2017). La importancia de tomar agua de buena calidad no se puede ignorar en los tiempos actuales, más aún cuando la contaminación de esta se ha convertido en un fenómeno inescapable. La mala calidad del agua potable y la contaminación de esta con microbios, ha llevado a que al menos la mitad de la población de países en desarrollo sufran de problemas de salud de vejiga, hígado, riñón, entre otros (Srivastav \& Kaur, 2020). La escasez de agua ha crecido a un ritmo alarmante, es así como 4.000 millones de habitantes, es decir, dos tercios de la población no tienen acceso al agua convirtiéndose así en una preocupación global .(United Nations Educational, Scientific and Cultural Organization, 2019a). Como respuesta a esta escasez se han desarrollado tecnologías de tratamiento de agua para asegurar la producción de agua de buena calidad y así cubrir la demanda de este recurso en condiciones aptas para el uso y/o consumo humano. Estas tecnologías pueden clasificarse en tres categorías principales: Físicas (Sedimentación, filtración, adsorción, rayos UV), químicas (Coagulación, electroquímica, oxidación, reducción catalítica, desinfección) y biológicas (Biodegradación biológica, digestores, biorreactores) (Ang \& Mohammad, 2019). Del grupo de tecnologías descritas anteriormente, la coagulación es uno de los procesos más antiguos que continúa siendo empleado en gran cantidad de plantas de tratamiento de agua residual y agua potable. La coagulación es un proceso que remueve las impurezas (Especialmente partículas en suspensión y coloides) del agua desestabilizando y aglomerando las partículas en agregados más grandes. Esto permite que el agregado se sedimente rápidamente para así poder separarlo fácilmente del agua (Jiang, 2015) . La coagulación es considerada como una de las alternativas más simples y costo-efectiva que puede acelerar la remoción de impurezas suspendidas en el agua. (Ang \& Mohammad, 2020) La gran demanda e interés en el proceso de 
coagulación ha fomentado la exploración de coagulantes de nueva generación con mejor desempeño y eficiencia. Esto incluye la búsqueda de coagulantes naturales como una alternativa de los de naturaleza química con el fin de apoyar los procesos sostenibles. A pesar de haber mostrado la eficiencia de los polímeros extraídos de plantas en un número substancial de artículos de investigación, tienen aún una poca aceptación y propagación en la industria del tratamiento de aguas.(Ang \& Mohammad, 2020).La principal ventaja de los coagulantes naturales es su renovabilidad, biodegradabilidad, no toxicidad y su buena relación costo beneficio. (Saleem et al. 2019). Los coagulantes naturales pueden ser derivados de los animales, las semillas, los crustáceos, de organismos microbianos y principalmente se destacan por contener mayores investigaciones a base de plantas vegetales y biomasa marina (Oladoja, 2015) El mecanismo de coagulación predominante en los coagulantes naturales difiere al de los de tipo inorgánicos y sintéticos, debido a que los naturales no forman precipitados de hidróxido en el agua. En cambio, debido a sus estructuras poliméricas cargadas con grupos funcionales, la neutralización de cargas y puenteo son los dos mecanismos dominantes para la formación de flóculos y la remoción de impurezas(Saleem \& Bachmann, 2019).En la actualidad los coagulantes distribuidos comercialmente son principalmente derivados de las conchas marinas (Chitosan) y plantas (Moringa y Tanina).(Graham et al., 2008). El resto de los coagulantes naturales no han sido comercializados y permanecen en pruebas de laboratorio o solamente son empleados en aplicaciones de pequeña escala 0 en casos específicos de investigación como por ejemplo las semillas de tamarindo, Moringa Oleífera, cascaras de plátano y cenizas volantes empleados como biocoagulantes (Nath et al., 2020). El principal obstáculo de los coagulantes naturales es la escases de estudios a escala industrial que permitan verificar el comportamiento en el tratamiento de agua potable y saber cuál es la dosificación necesaria(Hoa \& Hue, 2018). Para aumentar la confianza en los coagulantes naturales se han realizado investigaciones que buscan resaltar el gran desempeño de estos a través de: la aplicación de coagulantes naturales en procesos híbridos, es decir que, la mezcla entre dos coagulantes naturales mejora la extracción y la purificación del agua potable con respecto a las partículas suspendidas que necesitan ser removidas (Ang et al. 2020).

La seguridad hídrica es capaz de garantizar la calidad del agua bajo parámetros establecidos por las entidades gubernamentales y nacionales, además salvaguardar el acceso disponible a aquellas personas y animales que padecen de escases de agua, (United Nations Educational, Scientific and Cultural Organization, 2019), de igual forma protege el recurso hídrico de las actividades económicas y sociales de cada ser humano.

\section{METODOLOGIA}

Para llevar a cabo la presente investigación se ha realizado una amplia búsqueda de información sobre el tema del uso de coagulantes naturales en los procesos de potabilización del agua, mediante motores de búsqueda como Researchgate, Google Scholar, SciELO 
(Scientific Electronic Library Online), Dialnet, World Wide Science y Google Scholar. Complementando estas fuentes con reportes empresariales, informes de gestión empresariales o de gobierno y normatividad trabajada para los procesos de potabilización y consumo de agua, El filtro de la información se enfoca hacia la pertinencia del tema-fuente, numero de citaciones de las publicaciones y la experticia y/o trayectoria de los autores identificados. Otra de las herramientas claves de búsqueda fue mediante el empleo de las palabras claves descritas inicialmente en el presente artículo.

\section{ANALISIS Y DISCUSIÓN DE RESULTADOS}

El agua es una sustancia única y su característica principal es la capacidad de disolver una variedad de sustancias. Mientras el agua realiza su ciclo llamado ciclo hidrológico, entra en contacto con diversas substancias que pueden ser disueltas por el agua o ser suspendidas en esta. La cantidad y tipo de sustancias disueltas y suspendidas determinan la calidad del agua y si esta es apropiada o no para consumo humano (Schutte et al., 2006).

La contaminación en el agua es un problema común alrededor del mundo. Estos pueden ser de tipo geológicos o antropogénicos. (Fawell \& Nieuwenhuijsen, 2003). Altos niveles de contaminantes en el agua pueden causar efectos negativos en la salud humana, dependiendo de la susceptibilidad de cada individuo.(Sharma \& Bhattacharya, 2017) .

El tipo y concentración de los contaminantes naturales dependen de la geología por la cual fluye el agua, estos pueden llegar a estar en cantidades inaceptables contaminando así el recurso hídrico (Liu et al. 2005). Otros contaminantes son subproductos de actividades humanas tales como las industrias y la agricultura, estos son generalmente metales pesados como mercurio, cobre, cromo, plomo, y sustancias químicas peligrosas, como insecticidas y fertilizantes. Adicionalmente, un incorrecto almacenamiento $y$ disposición de químicos como pinturas, detergentes sintéticos, solventes, aceites, medicinas, desinfectantes, pesticidas, baterías, y combustibles pueden generar contaminación de aguas subterráneas. (Kass et al., 2005). Por tanto, toda el agua superficial y alguna subterránea requieren un tratamiento previo al consumo humano para que esta no represente un riesgo en la salud. Esta potencial afectación a la salud puede ser debido a la contaminación química, microbiológica, física o radioactiva. Sin embargo, la contaminación microbiológica es generalmente la más peligrosa para la salud humana.

La mayoría de los sistemas de tratamiento de agua están diseñados para remover la contaminación microbiológica y los contaminantes físicos que puedan promover la actividad microbiológica, es decir, los sólidos suspendidos en el agua. Adicionalmente casi siempre, se utiliza un desinfectante al final del proceso por dos grandes razones: primero inactiva cualquier remanente bacteriano como último paso del tratamiento, $y$, más importante aún provee un desinfectante residual el cual mata cualquier bacteria introducida durante el almacenamiento y la 
distribución (World Health Organization. Water, 2000).

En la actualidad, existen variedad de procesos de tratamiento de agua potable y su adecuación depende de la calidad del agua cruda en el proceso, nivel de entrenamiento del personal y recursos disponibles para la operación y mantenimiento. En la figura 1 se expone el procedimiento comúnmente utilizado para el proceso de potabilización de agua, además de que se explicará cada paso de manera general, profundizando en la etapa de coagulación.

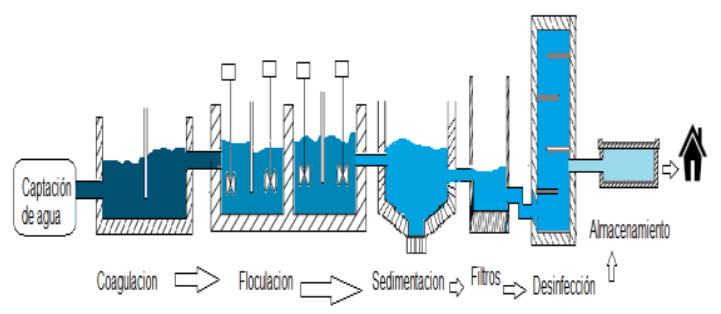

Figura 1. Elaboración propia Proceso de potabilización del agua

Finalmente, el proceso de potabilización de agua es importante para los seres humanos y juega un rol esencial en las necesidades diarias $y$ de esta forma previene posibles enfermedades.

El tratamiento de agua potable es esencial para la salud del ser humano y para la supervivencia de este, el recurso hídrico sin ningún proceso químico ni físico representa un riesgo para la salud humana ocasionando enfermedades a largo plazo. Debido al deterioro que presenta agua cruda por la intervención de varios factores industrias, agrícolas y domésticos entre otros(Abu Hasan et al., 2020).
El agua reporta diferentes tipos de contaminantes que se encuentran en las plantas potabilizadoras y los cuales se clasifican en físicos 0 propiedades organolépticas (color, olor sabor), químicos (Metales pesados, nitratos, fluoruros) y biológicos. (Bacterias, virus, protozoos) (Srivastav \& Kaur, 2020). Por este motivo se realizan varios tratamientos y se implementan nuevas tecnologías disminuyendo los impactos negativos que tienen los actuales procesos.

\subsection{Procesos para la potabilización de agua}

El tratamiento convencional en una planta de tratamiento de agua potable consiste en la transformación química, física y microbiología del recurso natural a través de procesos normalizados a lo largo del tiempo entre los cuales involucra las siguientes etapas: coagulación, floculación, sedimentación, filtración y desinfección (C.P. Gerba \& Pepper, 2019), En este orden de ideas el primero es la coagulación que se encarga de desestabilizar las partículas suspendidas del agua cruda, utilizando compuestos químicos como sales de aluminio, sulfato férrico y cloruro férrico (Maier et al., 2009), En segundo lugar, la floculación consiste en remover las partículas coloidales que se encuentran suspendidas y disueltas en el recurso hídrico, además elimina varios contaminantes presentes en la mezcla liquida, esta etapa es esencial para la formación de flóculos o flocs con alto peso molecular para que en el proceso se puedan aglomeran en diferentes tamaños (Ma et al., 2019), Un factor importante en la floculación es el potencial zeta, porque demuestra las interacciones presentes de las cargas electrostáticas, por otra parte, 
los floculantes se clasifican en químicos, sintéticos orgánicos y biofloculante (Xia et al., 2018), uno de los más utilizados es el poliacrilamida catiónica con alta viscosidad y densidad de carga (Ma et al., 2017).

Los procesos de coagulación y floculación van ligados de la mano, porque las impurezas desechadas en estas dos etapas son recolectadas en la tercera fase, que es la sedimentación. Este es un proceso físico que involucra un sistema de gravedad para suspender las partículas que son más densas que el agua y que causan la turbidez (Charles P. Gerba, 2009), gracias a esta etapa los lodos se dirigen al fondo de la superficie del sedimentador.

Como resultado del tratamiento anterior, la tercera etapa es la filtración que se encarga retener los residuos sólidos como la arena y la arcilla, utilizando carbón activado o antracita para adsorber los compuestos orgánicos presentes en el agua cruda (Hoslett et al., 2018), por tanto, el recurso hídrico finalmente tiene las características de ser incoloro y transparente (Charles P. Gerba, 2009). Después del proceso anterior se da lugar a la desinfección, el cual es un tratamiento indispensable en el planeta desde el siglo 20th y ha reducido los casos de enfermedades como la cólera y diarrea (Hu et al., 2018). Dicho de forma, en esta etapa se utiliza distintos métodos para la purificación del agua como la cloración, el dióxido de cloro y desinfección ultravioleta(Tang et al., 2020) con el propósito de eliminar los microrganismos, bacterias, y hongos presentes en la sustancia liquida incolora (Priya et al., 2020). Generalmente, las plantas potabilizadoras están regidas por normas y leyes con el objetivo de garantizar la calidad en el servicio de agua potable a los usuarios, por ejemplo, en Colombia está establecida la resolución del 2015 por el ministerio de salud y protección social. Finalmente, al llevar acabo cada una de las fases mencionadas, se almacena el agua tratada y se distribuye a los diferentes sectores económicos de la región.

\subsection{La coagulación}

En los procesos de potabilización, un coagulante es un producto químico, sintético, y natural que neutraliza las cargas negativas y desestabiliza las partículas suspendidas y disueltas, además es capaz de aglomerarse formando microflocs (Priya et al., 2020), cabe resaltar que se requiere una mezcla rápida para que el coagulante se disperse de manera adecuada sobre el líquido.

Generalmente, los coagulantes mas utilizados son los compuestos inorgánicos como el sulfato férrico y el sulfato de aluminio debido a su gran capacidad de desestabilizar las cargas electronegativas de las partículas, el segundo grupo, son los polímeros o coagulantes naturales que se derivan de plantas vegetales. En ambos casos, cuando el compuesto químico interactúa con agua, da lugar a diferentes mecanismos de reacción. Es decir, cuando el coagulante logra la atracción de las partículas, estas se adhieren a una doble capa y se comprimen, (Lozano-Rivas \& Lozano Bravo, 2015) y así mismo ocurre cuando se neutralizan y se adsorben las cargas negativas de los coloides para formar interacciones electrostáticas entre el 
coagulante metálico y los sólidos suspendidos(Freitas et al., 2018).

\subsubsection{Coagulantes naturales}

A lo largo del tiempo varios autores han investigado el comportamiento de los coagulantes a base de fuentes renovables como microorganismos, animales y plantas (Freitas et al., 2018) con el propósito de encontrar nuevas alternativas de desarrollo amigables con el medio ambiente. Además los polímeros naturales se han utilizado al transcurso de la historia humana por más de 2000 años en India, África, China, y México para tratar el agua cruda, gracias a las propiedades que se le confieren reducen la turbidez y el color del agua (Asrafuzzaman et al., 2011). Dicho lo anterior, a continuación, se describe en que consiste:

Los coagulantes polímeros son macromoléculas compuestas por unidades repetitivas de monómeros y son capaces de desestabilizar las partículas de un medio acuoso (Sillanpää et al., 2018) Generalmente son obtenidos de bacterias, animales y plantas (Moringa Oleífera, Opuntia ficus Indica, Strychnos potatorum Linn, Plantago ovate, Trigonella foenum graecum)(Saleem \& Bachmann, 2019). Estos polímeros naturales, son clasificados como catiónicas, aniónicas y no aniónicas. Es decir los polímeros catiónicas tienen carga positiva como el cloruro de dimetildialilamonio y dimetilamina, asimismo los elastómeros aniónicos se destacan por contener ácidos carboxílicos y finalmente se encuentra los no aniónicas que son solubles en el agua, estos incluyen poliacrilamidas de alto peso molecular (Bolto \& Gregory, 2007). Las principales propiedades de los polímeros es que no son tóxicos, son biodegradables, son recursos renovables, ecológicamente son amigables con el ambiente, tienen habilidades para encapsular materia orgánica e inorgánica, remueve y aglomera las impurezas presentes en los diferentes medios acuosos. (Renault et al., 2009)

\subsection{Aplicaciones}

Actualmente, los coagulantes extraídos de especies vegetales pueden ser aplicados en varias gamas de la industria, como por ejemplo, en el tratamiento de agua potable y residual mediante el uso de la Opuntia ficus Indica y la Moringa Oleífera( Taiwo et al., 2020). También, se emplea en la industria textil la goma de guar extraído del endospermo del frijol, para la formación de papel, como agente aglutinante para explosivos, en alimentos, productos farmacéuticos, y domésticos.(Pandey et al., 2014), Existe gran variedad extracciones de polisacárido vegetal por ejemplo la goma arábica extraído(árbol de acacia) goma tragacanto (Árbol astragalus), compuestos por Larabinosa, D-galactosa y se emplea como agente espesante, estabilizante (Nayak et al., 2018), además se encuentra el uso del tamarindo extraído de semillas del tamarindo indica es un polisacárido empleado para fabricar productos medicinales, estabilizados, aglutinante, nano fabricación de sistemas oculares vendaje de herida y otras que posee este polímero natural(Durai et al., 2012)

\subsection{Estudios de éxito de los coagulantes}

Estudios recientes, demuestran la capacidad que tienen los polímeros naturales en el tratamiento de agua 
potable y residual, la idea de ofrecer nuevas alternativas a los coagulantes ya existentes con excelentes resultados. Algunas de estas nuevas opciones son, el uso de semilla de nirmali y Eirchorrnia crassipes en aguas residuales producidas por las industrias textiles, para eliminar los sólidos disueltos y totales que se encuentran contenidos en los vertederos, utilizando dosis de $8-5 \mathrm{mg} / \mathrm{L}$ y logrando la reducción de turbidez en 53.85\% y 23.07\% respectivamente (Prabhakaran et al., 2020), Por otro lado se evalúa la eficiencia de las semillas de la Moringa Oleífera en una reserva de agua de Nigeria mediante una prueba de jarras, el cual disminuyo la turbidez en $58.18 \%$ y se demostró la capacidad antimicrobiana que tenia esta especie, cumple con los parámetros para ser consumida, ademas se observa que posee propiedades biocoagulantes y es biodegrable (Taiwo et al., 2020), la mayoría de revistas busca alternativas para clarificar el agua.

Por otro lado, se emplea el garbanzo (Cicer arietinum) y el frijol de Egipto (Dolichos lablab) como coagulante, utilizando $50 \mathrm{mg} / \mathrm{L}$ y $100 \mathrm{mg} / \mathrm{L}$ en un test de jarras, por consiguiente se logró reducir la turbidez del agua cruda en $95.89 \%$ y $88,9 \%$ respectivamente(Asrafuzzaman et al., 2011) . Por otra parte, se empleó la calabaza de hiedra, (Coccinia indica) que es una verdura rica en carbohidratos empleada para combatir la diabetes fiebre y lepra. Es este caso se realizó la extracción del mucilago de la fruta, para la eliminación de la turbidez del agua sintética, el cual fue efectiva con $94 \%$ en remoción; en adicción se comparó con el Quimbombó que es rico en proteínas y es comestible, De ahí que se logró remover las partículas suspendidas en $97.01 \%$ (S.
Y. Choy et al., 2015). No obstante, el autor, relato que se puede utilizar las siguientes legumbres en el tratamiento de agua y en otras aplicaciones lufa egipcia (Luffa cylindria), mani (arachis hypogaea), Glycine max, Lablad purpureus, y Phaseolus Angularis.

\subsection{Discusión}

Como se puede observar en este artículo, los coagulantes naturales cuentan con un papel prioritario en la investigación actual para su pronta implementación en los procesos de tratamiento de agua, esto debido a un aumento en la necesidad de integración de materiales biodegradables en todos los sectores industriales conforme pasa el tiempo, en virtud de aumentos en las restricciones y sanciones ambientales catalizadas por el calentamiento global y distintos tratados ambientales que se han llevado a cabo los últimos años. Sin embargo, dependiendo las condiciones del agua a tratar pueden obtenerse resultados inferiores comparando con los coagulantes inorgánicos usados en la actualidad. Por lo mismo, los coagulantes naturales aún tienen una serie de retos por superar y así poder lograr impactar el sector industrial de una manera significativa, por lo pronto se mencionarán algunos de los desafíos a los cuales se enfrentan.

Uno de los grandes desafíos que tienen los coagulantes naturales es la producción a escala industrial, debido al alto costo que se genera cuando se implementan, sin embargo, la efectividad de los polímeros extraídos es comprobada por números artículos donde se resalta la capacidad de reducir la turbidez de agua cruda a escala piloto, por tanto, este 
procedimiento surge como alternativa de reemplazo a los actuales coagulantes en los sectores industriales (Nath et al., 2020).

Como se mencionó anteriormente, se requiere buscar nuevas tecnologías verdes que sean sostenibles, innovadoras, viables tanto económica, ambiental y social que reduzcan la contaminación y los problemas que se enfrentan algunos compuestos químicos causando enfermedades a largo plazo (Oladoja, 2015). Por otra parte, uno de los retos más importantes es que los polímeros inorgánicos sean compatibles con otras tecnologías y al mismo tiempo sé requiere que provean de estabilidad y seguridad al sistema al cual van a hacer aplicado, en este caso se requiere que tenga poder de desestabilizar las partículas suspendidas en el tratamiento de agua potable. (Ang \& Mohammad, 2020)

Otro aspecto a tener en cuenta, es que los residuos generados por compuestos naturales sean biodegrable y se utilicen para el aprovechamiento de energía a partir de la materia orgánica que se retuvo (Sook Yan Choy et al., 2016), de igual forma, a futuro las tecnologías verdes deben ser estudias e implementadas para obtener los siguientes beneficios; ser amigables con el medio ambiente, reducir la dependencia de los productos químicos, menores costos en el tratamiento, bajo costo de adquisición de la materia prima, seguros para el consumo humano, y no corrosivos. (Sook Yan Choy et al., 2014). Sin embargo, el uso de algunos coagulantes naturales deteriora la vida útil de agua debido a las altas concentraciones de carbono orgánico disuelto (Saritha et al., 2019).

\section{CONCLUSIONES}

En relación a lo descrito y encontrado durante el desarrollo de este artículo, cabe resaltar la importancia del recurso hídrico en los ecosistemas, las actividades humanas y propiamente en la existencia del ser humano, siendo una de las primeras necesidades para su supervivencia, es así, como la preocupación por adquirir un agua de calidad para el consumo se vuele una prioridad para el mundo y con ello en la inversión de tiempo, investigación, presupuesto y tecnología en la implementación de técnicas para lograr el objetivo de unas condiciones aptas del recurso hídrico para las comunidades

El proceso de potabilización toma transcendencia al ser la principal herramienta para lograr el objetivo de propiedades adecuadas del agua para el consumo, por ende, cualquier insumo, operación o recurso que pueda optimizar este principio debe ser analizado y evaluado con la meta de buscar una calidad de vida y disminuir el actual o potencial estrés hídrico de algunas zonas del planeta.

Es así, como la implementación o desarrollo de coagulantes naturales en el proceso de Coagulación para la potabilización del agua es relevante puesto que está generando cambios ambientalmente sostenibles para el proceso y al mismo tiempo una responsabilidad con los resultados deseados de la calidad hídrica

Una de las etapas más importantes en el tratamiento de agua potable es la coagulación, porque se encarga de 
estabilizar la materia orgánica e inorgánica suspendida en el agua potable, esta fase generalmente se utiliza coagulantes inorgánicos, sin embargo, en este estudio se resalta el uso de los coagulantes derivados de fuentes vegetables, porque representan un gran avance en el desarrollo de la tecnología ambiental y sostenible, además representa mejores condiciones en la salud ambiental $y$ humana. Así mismo los coagulantes son eficientes en la reducción de los parámetros fisicoquímicos del agua cruda como el color, y la turbidez. Lo anterior es gracias a las siguientes características: al alto peso molecular que poseen, alta densidad de carga catiónica, facilidad de formar largas cadenas de polímeros, son ecológicos, no son tóxicos, y reducen el contenido de los lodos.

Como se mencionó en los casos de éxito todos lo coagulantes naturales son capaces de remover la turbidez de agua y participar en los mecanismos de reacción, finalmente cabe resaltar que se puede fabricar un polímero natural a través de plantas botánica, de la celulosa, de la goma(arabica, ghatti, tragacanto, karaya), mediante tubérculos como el almidón, la pectina, por medio de algas rojas, pardas y también de animales como la gelatina el quitosan la proteína de suero entre otros(Mirhosseini \& Amid, 2012).

\section{AGRADECIMIENTOS}

Agradecemos a la Fundación Universidad de América por el apoyo en el desarrollo de la presente revisión investigativa.

\section{REFERENCIAS BIBLIOGRAFÍCAS}

Abu Hasan, H., Muhammad, M. H., \& Ismail, N. 'Izzati. (2020). A review of biological drinking water treatment technologies for contaminants removal from polluted water resources. Journal of Water Process Engineering, 33, 101035. https://doi.org/10.1016/j.jwpe.2019.101035

Ahmadi, N., Chaibakhsh, N., \& Zanjanchi, M. A. (2016). Use of $D$ escurainia sophia $L$. As a natural coagulant for the treatment of dye-containing wastewater. Environmental Progress \& Sustainable Energy, 35(4), 996-1001. https://doi.org/10.1002/ep.12311

Ang, W. L., \& Mohammad, A. W. (2019). Integrated and hybrid process technology. En Sustainable Water and Wastewater Processing (pp. 279-328). Elsevier. https://doi.org/10.1016/B978-0-12-8161708.00009-0

Ang, W. L., \& Mohammad, A. W. (2020). State of the art and sustainability of natural coagulants in water and wastewater treatment. Journal of Cleaner Production, 121267.

https://doi.org/10.1016/j.jclepro.2020.1212 67

Asrafuzzaman, Md., Fakhruddin, A. N. M., \& Hossain, Md. A. (2011). Reduction of Turbidity of Water Using Locally Available Natural Coagulants. ISRN Microbiology, 2011 , 1-6. https://doi.org/10.5402/2011/632189

Bolto, B., \& Gregory, J. (2007). Organic polyelectrolytes in water treatment. Water Research, 41(11), 2301-2324. 
https://doi.org/10.1016/j.watres.2007.03.01 2

Choy, S. Y., Prasad, K. M. N., Wu, T. Y., \& Ramanan, R. N. (2015). A review on common vegetables and legumes as promising plant-based natural coagulants in water clarification. International Journal of Environmental Science and Technology, 12(1), 367-390. https://doi.org/10.1007/s13762-013-0446-2

Choy, Sook Yan, Prasad, K. M. N., Wu, T. Y., Raghunandan, M. E., \& Ramanan, R. N. (2014). Utilization of plant-based natural coagulants as future alternatives towards sustainable water clarification. Journal of Environmental Sciences, 26(11), 21782189.

https://doi.org/10.1016/j.jes.2014.09.024

Choy, Sook Yan, Prasad, K. N., Wu, T. Y., Raghunandan, M. E., \& Ramanan, R. N. (2016). Performance of conventional starches as natural coagulants for turbidity removal. Ecological Engineering, 94, 352364.

https://doi.org/10.1016/j.ecoleng.2016.05.0 82

Damkjaer, S., \& Taylor, R. (2017). The measurement of water scarcity: Defining a meaningful indicator. Ambio, 46(5), 513531. https://doi.org/10.1007/s13280-0170912-z

Durai, R., Rajalakshmi, G., Joseph, J., Kanchalochana, S., \& Hari, V. (2012). Tamarind seed polysaccharide: A promising natural excipient for pharmaceuticals. International Journal of Green Pharmacy, 6(4), 270. https://doi.org/10.4103/0973-8258.108205
Fawell, J., \& Nieuwenhuijsen, M. J. (2003). Contaminants in drinking water. British Medical Bulletin, 68(1), 199-208. https://doi.org/10.1093/bmb/ldg027

Food and Agriculture Organization of the United Nations. (2013). Afrontar la escasez de agua: Un marco de acción para la agricultura y la qeguridad alimentaria.

http://public.ebookcentral.proquest.com/ch oice/publicfullrecord.aspx?p=3239163

Freitas, T. K. F. S., Almeida, C. A., Manholer, D. D., Geraldino, H. C. L., de Souza, M. T. F., \& Garcia, J. C. (2018). Review of Utilization Plant-Based Coagulants as Alternatives to Textile Wastewater Treatment. En S. S. Muthu (Ed.), Detox Fashion (pp. 27-79). Springer Singapore. https://doi.org/10.1007/978981-10-4780-0_2

Gerba, Charles P. (2009). Drinking Water Treatment. En Environmental Microbiology (pp. 531-538). Elsevier. https://doi.org/10.1016/B978-0-12-3705198.00025-0

Gerba, C.P., \& Pepper, I. L. (2019). Drinking Water Treatment. En Environmental and Pollution Science (pp. 435-454).

Elsevier. https://doi.org/10.1016/B978-0-12-8147191.00024-0

Graham, N., Gang, F., Fowler, G., \& Watts, M. (2008). Characterisation and coagulation performance of a tannin-based cationic polymer: A preliminary assessment. Colloids and Surfaces A: Physicochemical and Engineering Aspects, $\quad 327(1-3), \quad$ 9-16. 
https://doi.org/10.1016/j.colsurfa.2008.05.0 45

Hoa, N. T., \& Hue, C. T. (2018). Enhanced water treatment by Moringa oleifera seeds extract as the bio-coagulant: Role of the extraction method. Journal of Water Supply: Research and Technology-Aqua. https://doi.org/10.2166/aqua.2018.070

Hoslett, J., Massara, T. M., Malamis, S., Ahmad, D., van den Boogaert, I., Katsou, E., Ahmad, B., Ghazal, H., Simons, S., Wrobel, L., \& Jouhara, H. (2018). Surface water filtration using granular media and membranes: A review. Science of The Total Environment, 639, 1268-1282. https://doi.org/10.1016/j.scitotenv.2018.05. 247

Hu, J., Chu, W., Sui, M., Xu, B., Gao, N., \& Ding, S. (2018). Comparison of drinking water treatment processes combinations for the minimization of subsequent disinfection by-products formation during chlorination and chloramination. Chemical Engineering Journal, 335, 352-361. https://doi.org/10.1016/j.cej.2017.10.144

Jiang, J.-Q. (2015). The role of coagulation in water treatment. Current Opinion in Chemical Engineering, 8, 36-44. https://doi.org/10.1016/j.coche.2015.01.00 8

Kass, A., Gavrieli, I., Yechieli, Y., Vengosh, A., \& Starinsky, A. (2005). The impact of freshwater and wastewater irrigation on the chemistry of shallow groundwater: A case study from the Israeli Coastal Aquifer. Journal of Hydrology, 300(1-4), 314-331. https://doi.org/10.1016/j.jhydrol.2004.06.01 3
Lozano-Rivas, W. A., \& Lozano Bravo, G. (2015). Potabilización del agua principios de diseño, control de proceses $y$ laboratorio. Universidad Piloto de Colombia.

http://search.ebscohost.com/login.aspx?dir ect=true \&scope $=$ site $\& \mathrm{db}=$ nlebk $\& A N=1593$ 375

Ma, J., Fu, K., Fu, X., Guan, Q., Ding, L., Shi, J., Zhu, G., Zhang, X., Zhang, S., \& Jiang, L. (2017). Flocculation properties and kinetic investigation of polyacrylamide with different cationic monomer content for high turbid water purification. Separation and Purification Technology, 182, 134143.

https://doi.org/10.1016/j.seppur.2017.03.0 48

Maier, R. M., Pepper, I. L., \& Gerba, C. P. (Eds.). (2009). Environmental microbiology (2nd ed). Elsevier/Academic Press.

Nath, A., Mishra, A., \& Pande, P. P. (2020). A review natural polymeric coagulants in wastewater treatment. Materials Today: Proceedings, S2214785320323324. https://doi.org/10.1016/j.matpr.2020.03.55 1

Nayak, A. K., Bera, H., Hasnain, M. S., \& Pal, D. (2018). Synthesis and Characterization of Graft Copolymers of Plant Polysaccharides. En Biopolymer Grafting: Synthesis and Properties (pp. 162). Elsevier. https://doi.org/10.1016/B9780-323-48104-5.00001-9

Odlare, M. (2014). Introductory Chapter for Water Resources. En Reference Module in Earth Systems and Environmental Sciences (p. B9780124095489090000). 
Elsevier. https://doi.org/10.1016/B978-012-409548-9.09035-7

Oladoja, N. A. (2015). Headway on natural polymeric coagulants in water and wastewater treatment operations. Journal of Water Process Engineering, 6, 174-192. https://doi.org/10.1016/j.jwpe.2015.04.004

Pandey, V. S., Verma, S. K., Yadav, M., \& Behari, K. (2014). Guar gum-g-N,N'dimethylacrylamide: Synthesis, characterization and applications. Carbohydrate Polymers, 99, 284-290. https://doi.org/10.1016/j.carbpol.2013.08.0 24

Prabhakaran, G., Manikandan, M., \& Boopathi, M. (2020). Treatment of textile effluents by using natural coagulants. Materials Today: Proceedings, S2214785320318034.

https://doi.org/10.1016/j.matpr.2020.03.02 9

Priya, T., Mishra, B. K., \& Prasad, M. N. V. (2020). Physico-chemical techniques for the removal of disinfection by-products precursors from water. En Disinfection $\mathrm{By}$ products in Drinking Water (pp. 23-58). Elsevier. https://doi.org/10.1016/B978-008-102977-0.00002-0

Renault, F., Sancey, B., Badot, P.-M., \& Crini, G. (2009). Chitosan for coagulation/flocculation processes - An eco-friendly approach. European Polymer Journal, $\quad 45(5)$, 1337-1348. https://doi.org/10.1016/j.eurpolymj.2008.12 .027

Saleem, M., \& Bachmann, R. T. (2019). A contemporary review on plant-based coagulants for applications in water treatment. Journal of Industrial and Engineering Chemistry, 72, 281-297. https://doi.org/10.1016/j.jiec.2018.12.029

Saritha, V., Karnena, M. K., \& Dwarapureddi, B. K. (2019). "Exploring natural coagulants as impending alternatives towards sustainable water clarification" - A comparative studies of natural coagulants with alum. Journal of Water Process Engineering, 32, 100982. https://doi.org/10.1016/j.jwpe.2019.100982

Schutte, F., South Africa, \& Water Research Commission. (2006). Handbook for the operation of water treatment works. Water Research Commission.

Sharma, S., \& Bhattacharya, A. (2017). Drinking water contamination and treatment techniques. Applied Water Science, $\quad 7(3), \quad$ 1043-1067. https://doi.org/10.1007/s13201-016-0455-7

Sillanpää, M., Ncibi, M. C., Matilainen, A., \& Vepsäläinen, M. (2018). Removal of natural organic matter in drinking water treatment by coagulation: A comprehensive review. Chemosphere, 190, 54-71. https://doi.org/10.1016/j.chemosphere.201 7.09.113

Srivastav, A. L., \& Kaur, T. (2020a). Factors affecting the formation of disinfection by-products in drinking water: Human health risk. En Disinfection Byproducts in Drinking Water (pp. 433-450). Elsevier. https://doi.org/10.1016/B978-008-102977-0.00019-6

Srivastav, A. L., \& Kaur, T. (2020b). Factors affecting the formation of disinfection by-products in drinking water: 
Human health risk. En Disinfection Byproducts in Drinking Water (pp. 433-450). Elsevier. https://doi.org/10.1016/B978-008-102977-0.00019-6

Taiwo, A. S., Adenike, K., \& Aderonke, O. (2020). Efficacy of a natural coagulant protein from Moringa oleifera (Lam) seeds in treatment of Opa reservoir water, Ile-Ife, Nigeria. Heliyon, 6(1), e03335. https://doi.org/10.1016/j.heliyon.2020.e033 35

Tang, Y., Long, X., Wu, M., Yang, S., Gao, N., Xu, B., \& Dutta, S. (2020). Bibliometric review of research trends on disinfection by-products in drinking water during 19752018. Separation and Purification Technology, 241, 116741. https://doi.org/10.1016/j.seppur.2020.1167 41

Tejado Gallegos, M., \& Olmos Pérez, A. (2014). El derecho humano al agua potable y saneamiento.

Una Ryan: On the Importance of Clean Potable Water. (2017). Trends in Parasitology, 33(7), 489-490. https://doi.org/10.1016/j.pt.2017.03.007

United Nations Educational, Scientific and Cultural Organization. (2019a). Informe Mundial de Naciones Unidas sobre el Desarrollo de los Recursos Hídricos 2019:
No dejar a nadie atrás. UN. https://doi.org/10.18356/e96937a1-es

United Nations Educational, Scientific and Cultural Organization. (2019b). Informe Mundial de Naciones Unidas sobre el Desarrollo de los Recursos Hídricos 2019: No dejar a nadie atrás. UN. https://doi.org/10.18356/e96937a1-es

World Health Organization. Water, S. and H. T. (2000). WHO guidelines for drinking water quality: Training pack. WHO Seminar Pack for Drinking-Water Quality. $\mathrm{WHO}$ IRIS. https://apps.who.int/iris/handle/10665/6621 8

Xia, X., Lan, S., Li, X., Xie, Y., Liang, Y., Yan, P., Chen, Z., \& Xing, Y. (2018). Characterization and coagulationflocculation performance of a composite flocculant in high-turbidity drinking water treatment. Chemosphere, 206, 701-708. https://doi.org/10.1016/j.chemosphere.201 8.04.159 